\title{
Hyaluronic Acid/Polylysine Composites for Local Drug Delivery: A Review
}

\author{
Eliza Tracuma ${ }^{1, a}$ and Dagnija Loca ${ }^{1, b^{*}}$ \\ ${ }^{1}$ Rudolfs Cimdins Riga Biomaterials Innovations and Development Centre of RTU, Institute of \\ General Chemical Engineering, Faculty of Materials Science and Applied Chemistry, Riga \\ Technical University, Pulka 3, Riga, Latvia, LV-1007 \\ aeliza.tracuma@rtu.Iv, bdagnija.loca@rtu.Iv
}

Keywords: hyaluronic acid, polylysine, drug delivery, multilayer film, polyelectrolyte complex.

\begin{abstract}
Site specific drug delivery systems (DDS) are usually developed to overcome the side effects of conventional ones (e.g. injections or oral ingestions). Creating site specific DDS means creating smart drug delivery vehicles characterized by greater efficiency, safety, predictable therapeutic response as well as controlled and prolonged drug release periods. DDS made of hyaluronic acid (HA) and poly-L-lysine (PLL) are promising candidates in the field of local drug delivery due to their high biocompatibility. Moreover, electrostatic attractions between negatively charged HA and positively charged PLL can be used to fabricate multilayer films, bilayer films, and hydrogels, avoiding the application of toxic crosslinking agents. In this review, we report the preparation of HA/PLL composites exploiting their intrinsic properties, as well as application possibilities of the developed composite as controlled drug delivery systems in bone tissue, central nervous system, and gene engineering.
\end{abstract}

\section{Introduction}

HA is one of the most often used biopolymers due to its non-immunogenicity, versatility, biocompatibility, and biofunctionality. $\mathrm{HA}$ is a linear anionic polysaccharide; it is a major component of an extracellular matrix and plays an important role to maintain the viscoelasticity of tissues, its mechanical integrity, homeostasis, and lubrication [1,2]. In the human body HA is widely found in epithelial tissues, synovial fluid, umbilical cord, joints, pleura, tendons, and sheaths [3]. Recent investigations of HA applications in the biomedical field include tissue engineering, cell therapy, drug delivery, and regenerative medicine [1].

Since HA is not able to form gels without modification, several strategies have been applied to modify HA-based composites for biomedical purposes, in particular, for drug delivery [1]. Recent studies have shown a high biocompatibility of systems made combining HA and PLL [4]. PLL is polycationic polyamino acid with a wide range of antimicrobial spectrum and has been used as a scaffold for drug/gene delivery due to its cationic properties [5]. The electrostatic attractions between negatively charged carboxyl group of HA $(\mathrm{pKa}=2.9)$ and positively charged primary amine groups of PLL $(\mathrm{pKa}=10.5)$ can be utilized to fabricate films, polyelectrolyte complexes, and hydrogels with potential for drug delivery (see Fig. 1) [6, 7].

\section{HA/PLL Multilayer Films for Drug Delivery}

Combination of HA/PLL biopolymers has been successfully assembled into (HA/PLL)n polyelectrolyte multilayer films by the layer-by-layer $(\mathrm{LbL})$ electrostatic technique. The basic principle of preparation of such film is that the repeated electrostatic adsorption of positively and negatively charged polymer onto a charged substrate [8]. The LbL technique allows to coat biomaterial surfaces with a thin polymeric layer [4]. For example, LbL multilayer film could be constructed on medical implants with drug delivery function to increase local drug concentration, control local drug release, and prevent bacterial colonization around the device $[9,10]$. Important advantage of HA/PLL multilayer coatings is their antibacterial properties - inhibiting the growth of gram-positive bacteria Staphylococcus aureus and gram-negative bacteria Pseudomonas aeruginosa 
and Escherichia coli [11]. Moreover, HA/PLL multilayer films can be loaded with drugs and other molecules either through chemical attachment of drugs to HA or PLL [9] or by loading drugs directly onto the fabricated multilayer [12]. This makes HA/PLL multilayer films attractive for local drug delivery applications [4].

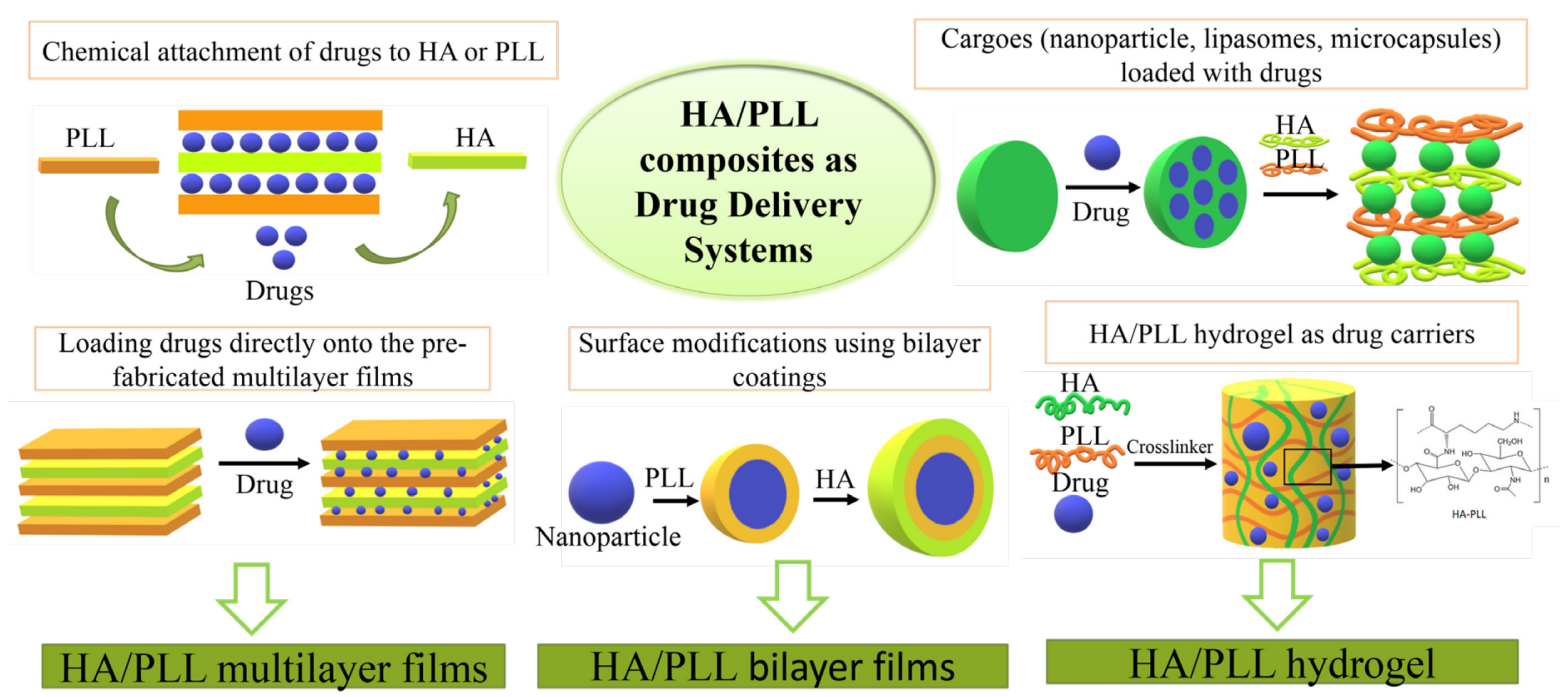

Fig.1. HA/PLL composites as drug delivery systems.

HA/PLL are exponentially growing films, which are thicker compared to the linearly growing ones [8,13-15]. Multilayer films can reach micrometer dimensions and act as reservoirs for biomolecules [6]. Therefore, HA/PLL films have enough capacity for small drug molecules and have been loaded with: an antibiotic - gentamicin, anticancer drugs - paclitaxel and doxorubicin, and an anti-inflammatory drug - diclofenac $[6,12]$. Furthermore, DNA, peptides, larger colloidal structures, and various biomolecule containing carriers such as microcapsules, liposomes [10, 16], and nanoparticles [15] can be entrapped in such films and released by external stimulation [13-15].

HA and PLL have been classified as weak polyelectrolytes; their physicochemical properties can be affected by $\mathrm{pH}$ and ionic strength of the surrounding medium [17]. Recent work of Hellwig et al. [4] showed the differences between native films (made by electrostatic attractions) and fully crosslinked films (crosslinked using 1-ethyl-3-(3-dimethylamino-propyl) carbodiimide (EDC)) in response to external stimuli. Native HA/PLL films behave like physical gels, and any environmental changes decrease their mechanical stability, making them viable for local drug delivery systems. Fully crosslinked HA/PLL films behave like stiff chemical gels, and can be used as resistant coatings for medical devices [4].

Many new strategies have been developed to improve HA/PLL multilayer films for drug delivery. Recently, Schmidt et al. [15] and Kohler et al. [18] suggested to coat a HA/PLL film with $5 \mathrm{~nm}$ thin gold nanoparticle layer to increase their stiffness and improve the cell adhesion. Based on the previous studies, Prokpovic et al. [14] investigated gold coated HA/PLL films for drug delivery applications. The obtained results indicated that gold layer acts as a semipermeable membrane, which allows small molecules diffuse freely out of the coated film. These gold coated films allow to control the release of film-loaded biomolecules [14].

Another strategy to achieve controlled drug delivery is to load HA/PLL films with drug containing carriers, such as liposomes. Liposomes are unstable in contact with solid surfaces like polyelectrolyte films. Therefore, Volodkin et al. reported that liposomes can be stabilized and protected by PLL coating and then loaded in HA/PLL films $[10,16]$. Some studies have reported the interactions between cationic protein lysozyme (Lys) and HA/PLL films. High Lys loading capacity was observed, which allowed to make HA/PLL films with high internal Lys concentration [13].

Furthermore, HA/PLL multilayer films have a potential use in bone tissue regeneration approaches. HA/PLL films have shown efficient properties for delivery of growth factors, such as 
bone morphogenetic protein 2 (BMP-2). BMP-2 loaded HA/PLL multilayer films can retain tunable quantities of BMP-2, delivering protein in different concentration levels and inducing the cell differentiation to osteoblasts [17, 19].

$\mathrm{pH}$-sensitive drug release from HA/PLL multilayers is another drug delivery strategy. By attaching doxorubicin to HA/PLL multilayers with a covalent hydrazone bond, it is possible to release the drug in a controlled way by bond breakdown in the acidic environment of tumor tissue $(\mathrm{pH}=5.2-7.2)[9]$.

\section{HA/PLL Polyelectrolyte Bilayer Films for Drug Delivery}

Polyelectrolyte complex (PECs) bilayer systems (HA/PLL) 2 are composed of two polymer layers on a charged surface. PECs have a potential in gene/drug carrier development and tissue engineering applications.

In gene engineering HA and PLL biopolymer complexes can be used as vehicles for magnetic hyperthermia-based cancer therapy. Superparamagnetic iron oxide nanoparticles can be encapsulated in modified HA micelles, followed by their coating with positively charged PLL as DNA-carrier. Afterwards, PLL can be complexed with negatively charged DNA, which then can be delivered exactly into cells [20]. Furthermore, HA/PLL multilayer films have a potential in bone tissue regeneration approaches. Recent work of Amorim et al. showed that a HA/PLL multilayer coating onto charged surfaces of $\mathrm{SiO}_{2}$ can induce osteogenic differentiation of human bone marrow stem cells at low concentrations [7], while Dreaden et al. [21] described responsive bilayers in tumor environment. HA/PLL drug carriers actively target tumors through their interactions with target cancer stem cell receptor $\mathrm{CD} 44$ at hypoxic tumor $\mathrm{pH}$

PECs phenomenon can also be used to control drug delivery from HA microgels loaded with hydrophilic drug through building an impermeable PLL shell around the gel. This property allows both to protect the HA microgel from enzymatic degradation and to encapsulate proteins and peptides with non-affinity to the matrix, like insulin and dextran. Herein the concept of drug delivery is to lock hydrophilic drugs inside a HA/PLL core [22]. Recently, Pan et al. developed HA/PLL complexes forming a tunable core-corona structure, where HA/PLL served as a core and HA chains were used as corona. Possible modifications of such structures make these complexes potential candidates for the development of controlled drug or gene delivery vehicles [23].

\section{HA/PLL Hydrogels for Drug Delivery}

Polymeric hydrogels are commonly used for local drug delivery; however, little attention has been paid to HA/PLL hydrogel systems. The application of HA/PLL hydrogels have been generally studied in context of central nervous system (CNS) and bone/brain tissue engineering.

Hydrogel-like particles called nanogels are composed of peptide-polysaccharide composites and represent a new class of bioresponsive carriers with tunable swelling and drug release profiles. Recently Lawanprasert et al. developed non-covalently crosslinked HA/PLL nanogels loaded with small molecules vancomycin hydrochloride and doxorubicin hydrochloride [24].

HA/PLL hydrogels containing curcumin and BMP-2 can be used as biomaterial scaffolds for bone tissue regeneration. Scaffolds of HA/PLL hydrogels are formed by amide bonds via condensation reaction between biopolymers using 4-(4,6-dimethoxy-1,3,5-triazin-2-yl)-4-methyl morpholinium chloride (DMT-MM) as a condensation agent [25]. Another approach is to modify HA hydrogels with PLL and specific growth factor receptors. HA is crosslinked by complexation of carboxyl groups with PLL using adipic dihydrazide (ADH) or EDC as crosslinkers [26-29]. Hydrogel antibody delivering systems have a potential in CNS injury treatment for a prolonged period of time $[27,28]$. For spinal cord injury such hydrogel modifications stimulate the formation of glial scar, supporting angiogenesis and promoting axonal extensions.

HA/PLL copolymer hydrogels have a potential as scaffolds for brain tissue deffect repair in brain tissue engineering. For this purpose HA and PLL are chemically crosslinked with EDC, obtaining hydrogels with porous structure and viscoelastic properties similar to the neural tissues [30]. 
A number of strategies have been developed for HA modification mainly through chemical modifications using crosslinkers. Synthesis of HA based hydrogels requires highly alkaline or acidic environments, making difficult to incorporate sensitive drug molecules and living cells into the hydrogels [31]. In biomedicine, preference is given to physically crosslinked hydrogels, thus avoiding potential toxicity of unreacted chemical crosslinkers, organic solvents, and chemical reagents $[32,33]$.

\section{Conclusions}

Systems composed of hyaluronic acid and poly-L-lysine are promising biomaterials. They can be modified with drugs, nanoparticles, and microcapsules to serve as local and controlled drug delivery tools. Such systems could be used for bone regeneration, cell/gene transport, tissue engineering, surface modifications, and cancer treatment (see Table 1). In contrast to multilayer films and polyelectrolyte complexes, little attention has been paid to hyaluronic acid and poly-L-lysine hydrogels and their potential as drug delivery vehicles. As multilayer films and polyelectrolyte complexes composed of hyaluronic acid and poly-L-lysine can be formed without chemical crosslinkers, similar approaches also could be applied to hyaluronic acid and poly-L-lysine hydrogel development, focusing on "green" (using nontoxic crosslinkers) hyaluronic acid and polyL-lysine hydrogel synthesis methods, intended for the local drug delivery in various biomedical applications.

Table 1. Hyaluronic acid and poly-L-lysine composite for biomedical application

\begin{tabular}{|c|c|c|c|}
\hline $\begin{array}{l}\text { Hyaluronic acid and } \\
\text { poly-L-lysine } \\
\text { composites }\end{array}$ & Biomedical application & Active substances & References \\
\hline \multirow{5}{*}{ Multilayer films } & Controlled drug delivery & Gold nanoparticles & {$[14,15,18]$} \\
\hline & $\begin{array}{l}\text { Biocompatible coatings with } \\
\text { effective local drug delivery } \\
\text { for biomedical devices }\end{array}$ & Liposomes & {$[10,16]$} \\
\hline & Bone tissue regeneration & Bone morphogenetic protein 2 & {$[17,19]$} \\
\hline & $\begin{array}{l}\mathrm{pH} \text {-sensitive drug release for } \\
\text { tumor treatment }\end{array}$ & Doxorubicin & [9] \\
\hline & $\begin{array}{l}\text { Surface coatings with drug } \\
\text { delivery ability }\end{array}$ & Paclitaxel & {$[12]$} \\
\hline \multirow{3}{*}{ Bilayer films } & Gene engineering & $\begin{array}{c}\text { Superparamagnetic iron oxide } \\
\text { nanoparticles }\end{array}$ & {$[20]$} \\
\hline & Bone tissue regeneration & Silicon dioxide & [7] \\
\hline & Controlled drug delivery & Insulin and dextran & [22] \\
\hline \multirow{3}{*}{ Hydrogels } & $\begin{array}{l}\text { Drug resistant and sensitive } \\
\text { cancer cell treatment }\end{array}$ & $\begin{array}{l}\text { Vancomycin hydrochloride and } \\
\text { doxorubicin hydrochloride }\end{array}$ & {$[24]$} \\
\hline & Bone tissue regeneration & Bone morphogenetic protein 2 & {$[25]$} \\
\hline & $\begin{array}{l}\text { Central nervous system } \\
\text { engineering }\end{array}$ & Nogo-66 receptor antibody & {$[27,28]$} \\
\hline
\end{tabular}

\section{Acknowledgement}

The authors acknowledge financial support from the Latvian Council of Science research project No. 1zp-2019/1-0005 "Injectable in situ self-crosslinking composite hydrogels for bone tissue regeneration (iBone)". 


\section{References}

[1] S. Trombino, C. Servidio, F. Curcio, R. Cassano, Strategies for Hyaluronic Acid-Based Hydrogel Design in Drug Delivery, Pharmaceutics. 11 (2019) 1-17.

[2] X. Xu, A.K. Jha, D.A. Harrington, M.C. Farach-Carson, X. Jia, Hyaluronic acid-based hydrogels: from a natural polysaccharide to complex networks, Soft Matter. 8 (2012) 3280-3294.

[3] N.M. Salwowska, K.A. Bebenek, D.A. Zadlo, D.L. Wcislo-Dziadecka, Physiochemical properties and application of hyaluronic acid: a systematic review, J. Cosmet. Dermatol. 15 (2016) $1-7$.

[4] J. Hellwig, J. Strebe, R. Klitzing, Effect of environmental parameters on the nano mechanical properties of hyaluronic acid/poly(l-lysine) multilayers, Phys. Chem. Chem. Phys. 20 (2018) 1908219086.

[5] S.C. Shukla, A. Singh, A.K. Pandey, A. Mishra, Review on production and medical applications of e-polylysine, Biochem. Eng. J. 65 (2012) 70-81.

[6] V.Z. Prokopovi, C. Duschl, D. Volodkin, Hyaluronic Acid/Poly-L-Lysine Multilayers as Reservoirs for Storage and Release of Small Charged Molecules, Macromol. Biosci. 15 (2015) 1357-1363.

[7] S. Amorim, A. Martins, N.M. Neves, L. Reis, R.A. Pires, Hyaluronic acid/poly-L-lysine bilayered silica nanoparticles enhance the osteogenic differentiation of human mesenchymal stem cells, J. Mater. Chem. 2 (2014) 6939-6946.

[8] S.E. Burke, C.J. Barrett, pH-Responsive Properties of Multilayered Poly(L-lysine )/Hyaluronic Acid Surfaces, Biomacromolecules. 4 (2003) 1773-1783.

[9] L. Wang, K. Ren, H. Wang, Y. Wang, J. Ji, pH-sensitive controlled release of doxorubicin from polyelectrolyte multilayers, Colloids Surf. B Biointerfaces. 125 (2015) 127-133.

[10] D. V Volodkin, P. Schaaf, H. Mohwald, J. Voegel, V. Ball, Effective embedding of liposomes into polyelectrolyte multilayered films: the relative importance of lipid-polyelectrolyte and interpolyelectrolyte interactions, Soft Matter. 5 (2009) 1394-1405.

[11] D. Alkekhia, A. Shukla, Influence of poly-l-lysine molecular weight on antibacterial efficacy in polymer multilayer films, J. Biomed. Mater. Res. A. 107 (2019) 1324-1339.

[12] C. Vodouhe, E. Le Guen, J.M. Garza, G. Francius, C. Dejugnat, J. Ogier, P. Schaaf, J.C. Voegel, P. Lavalle, Control of drug accessibility on functional polyelectrolyte multilayer films, Biomaterials. 27 (2006) 4149-4156.

[13] N. Velk, K. Uhlig, A. Vikulina, C. Duschl, D. Volodkin, Mobility of Lysozyme in Poly(Llysine)/Hyaluronic Acid Multilayer Films, Colloids Surf. B Biointerfaces. 147 (2016) 343-350.

[14] V.Z. Prokopovic, C. Duschl, D. V. Volodkin, Hyaluronic acid/poly-L-lysine multilayers coated with gold nanoparticles: Cellular response and permeability study, Polym. Adv. Technol. 25 (2014) 1342-1348.

[15] S. Schmidt, N. Madaboosi, K. Uhlig, D. Ko, A. Skirtach, C. Duschl, H. Mo, D. V Volodkin, Control of Cell Adhesion by Mechanical Reinforcement of Soft Polyelectrolyte Films with Nanoparticles, Langmuir. 28 (2012) 7249-7257.

[16] D. Volodkin, Y. Arntz, P. Schaaf, H. Moehwald, J. Voegel, V. Ball, Composite multilayered biocompatible polyelectrolyte films with intact liposomes: stability and temperature triggered dye release, Soft Matter. 4 (2008) 122-130.

[17] T. Crouzier, L. Fourel, T. Boudou, C. Albiges-rizo, C. Picart, Presentation of BMP-2 from a Soft Biopolymeric Film Unveils its Activity on Cell Adhesion and Migration, Adv. Mater. 3 (2011) 111-118. 
[18] D. Kohler, N. Madaboosi, M. Delcea, S. Schmidt, B.G. De Geest, D. V Volodkin, H. Mohwald, A.G. Skirtach, Patchiness of Embedded Particles and Film Stiffness Control Through Concentration of Gold Nanoparticles, Adv. Mater. 24 (2012) 1095-1100.

[19] F. Gilde, R. Guillot, J.F. Mano, D. Logeart-avramoglou, Secondary Structure of rhBMP - 2 in a Protective Biopolymeric Carrier Material, Biomacromolecules. 13 (2012) 2-8.

[20] M. Muthiah, M. Moon, Y.Y. Jeong, SPION Loaded Poly(L-lysine)/Hyaluronic Acid Micelles as MR Contrast Agent and Gene Delivery Vehicle for Cancer Theranostics, Macromol. Res. 25 (2017) 446-451.

[21] E.C. Dreaden, S.W. Morton, K.E. Shopsowitz, J. Choi, Z.J. Deng, N. Cho, P.T. Hammond, Bimodal tumor-targeting from microenvironment responsive hyaluronan layer-by-layer (LbL) nanoparticles, ACS Nano.8 (2014) 8374-8382.

[22] H. Labie, A. Perro, V. Lapeyre, B. Goudeau, R. Auzely, V. Ravaine, Sealing hyaluronic acid microgels with oppositely-charged polypeptides : a simple strategy for packaging hydrophilic drugs with on-demand release, J. Colloid Interface Sci. 535 (2019) 16-27.

[23] W. Pan, D. Yin, H. Jing, H. Chang, H. Wen, D. Liang, Core-Corona Structure Formed by Hyaluronic Acid and Poly(L-lysine) via Kinetic Path, Chinese J. Polym. Sci. 37 (2019) 36-42.

[24] A.W. Simonson, A. Lawanprasert, T.D.P. Goralski, K.C. Keiler, S.H. Medina, Bioresponsive Peptide-Polysaccharide Nanogels - A Versatile Delivery System to Augment the Utility of Bioactive Cargo, Nanomedicine. 17 (2019) 391-400.

[25] E. Kim, S.J. Yoon, K. Noh, D. Lee, Dual Effect of Curcumin/BMP-2 Loaded in HA/PLL Hydrogels on Osteogenesis In Vitro and In Vivo, J. Nanosci. Nanotechnol. 17 (2017) 143-152.

[26] Y. Wei, Y. He, C. Xu, Y. Wang, B. Liu, X. Wang, X. Sun, F. Cui, Q. Xu, Hyaluronic acid hydrogel modified with nogo-66 receptor antibody and poly-L-lysine to promote axon regrowth after spinal cord injury, J. Biomed. Mater. Res. B Appl. Biomater. 95 (2010) 110-117.

[27] L. Pan, Y. Ren, F. Cui, Q. Xu, Viability and Differentiation of Neural Precursors on Hyaluronic Acid Hydrogel Scaffold, J. Neurosci. Res. 87 (2009) 3207-220.

[28] Y.T. Wei, X.D. Sun, X. Xia, F.Z. Cui, Y. He, B.F. Liu, Q.Y. Xu, Hyaluronic Acid Hydrogel Modified with Nogo-66 Receptor Antibody and Poly(L-Lysine) Enhancement of Adherence and Survival of Primary Hippocampal Neurons. J. Bioact. Compat. Polym. 24 (2009) 205-219.

[29] Y. Li, H. Yu, H. Huang, M. Guo, R. Hua, The hetero-transplantation of human bone marrow stromal cells carried by hydrogel unexpectedly demonstrates a significant role in the functional recovery in the injured spinal cord of rats, Brain. Res. 1634 (2015) 1-13.

[30] W.M. Tian, S.P. Hou, J. Ma, C.L. Zhang, Q.Y. Xu, I.S. Lee, H.D. Li, M. Spector, F.Z. Cui, Hyaluronic Acid-Poly-D-Lysine-Based Three-Dimensional Hydrogel for Traumatic Brain Injury, Tissue Eng. 11 (2005) 513-525.

[31] S.K. Hahn, A.S. Hoffman, Preparation and characterization of biocompatible polyelectrolyte complex multilayer of hyaluronic acid and poly-L-lysine, Int. J. Biol. Macromol. 37 (2005) 227231.

[32] C. Vasile, D. Pieptu, R.P. Dumitriu, R. Academy, Chitosan/hyaluronic acid polyelectrolyte complex hydrogels in the management of burn wounds. Rev. Med. Chir. Soc. Med. Nat. Iasi. 117 (2013) 565-571.

[33] E.A. Kamoun, E.R.S. Kenawy, X. Chen, A review on polymeric hydrogel membranes for wound dressing applications: PVA-based hydrogel dressings, J. Adv. Res. 8 (2017) 217-233. 